\title{
Self-organizing Visualization and Pattern Matching of Vectorcardiographic QRS Waveforms
}

\author{
Hui Yang ${ }^{1, *}$ and Fabio Leonelli ${ }^{2}$ \\ ${ }^{1}$ Complex Systems Monitoring, Modeling and Control Laboratory \\ The Pennsylvania State University, University Park, PA, USA \\ ${ }^{2}$ Cardiology Department \\ James A. Haley Veterans' Hospital, Tampa, FL 33620 USA.
}

*Corresponding author:

Hui Yang, e-mail: huy25@ @su.edu; voice: (814) 865-7397; Fax: (814) 863-4745

(C) 2016. This manuscript version is made available under the Elsevier user license http://www.elsevier.com/open-access/userlicense/1.0/ 


\begin{abstract}
QRS morphology is commonly used in the electrocardiographic diagnosis of ventricular depolarization such as left bundle branch block (LBBB) and ventricular septal infarction. We investigated whether pattern matching of QRS loops in the 3-dimensional vectorcardiogram (VCG) will improve the grouping of patients whose space-time electrical activity akin to each other, thereby assisting in clinical decision making. First, pattern dissimilarity of VCG QRS loops is qualitatively measured and characterized among patients, resulting in a $93 \times 93$ distance matrix of patient-to-patient dissimilarity. Each patient is then represented as a node in the network (or a star in the galaxy), but node locations are optimized to preserve the dissimilarity matrix. The optimization is achieved with a self-organizing algorithm that iteratively minimizes the network energy. Experimental results showed that patients' locations converge as the representation error reaches a stable phase. The convergence is independent of initial locations of network nodes. Most importantly, 93 patients are automatically organized into 3 clusters of healthy control, LBBB, and infarction. Spatial coordinates of nodes (or patients) are evidently novel predictors that can be used in the computer-assisted detection of cardiac disorders. Selforganizing pattern matching is shown to have strong potentials for large-scale unsupervised learning of patient groups.
\end{abstract}

Keywords: vectorcardiography, QRS waveforms, pattern matching, self-organizing network 


\section{INTRODUCTION}

The Electrocardiogram remains the most commonly performed cardiac test, which is essential to the diagnosis and management of a large number of cardiac pathologies. Given the large number of ECG acquired daily, computerized interpretation of tracings can be extremely time-saving and conducive to clinical decision support [1]. For this reason, automated computer programs have nowadays become a fixed feature in the great majority of commercially available ECG devices. As the computer diagnostic accuracy of interpretation is often considered too low, the majority of ECGs will be over read by a qualified physician [2]. The increasing mismatch between competent cardiologists and the increasing number of ECGs obtained has generated a number of dissenting opinions [3,4] including Guidelines recommendations [5] where automated interpretation of ECG is part of the patients' initial assessment. Existing ECG algorithms mainly focus on a time-scale representation of cardiac electrical events ignoring a large amount of information contained in the spatial distribution of the ECG. Therefore, there is a need to study new vectorcardiographic methods to examine the dissimilarity of space-time electrical activity in the heart.

Among many cardiac pathologies, left bundle branch block (LBBB) is an electrocardiographic diagnosis based on QRS patterns such as time duration, morphology, notching or slurring. Such diagnostic criteria play important roles in the selection of LBBB patients for cardiac resynchronization therapy (CRT). It is worth mentioning that QRS area of 3lead VCG was recently shown to effectively predict the CRT responses and equivalently perform as well as the state-of-the-art ECG definition of LBBB [6]. The effectiveness of VCG for the optimization of CRT therapy was demonstrated in the experimental study of canine left bundle branch block hearts [7]. In addition, the VCG feature of QRS-T area angle was shown to effectively predict sudden cardiac death after acute coronary syndromes [8]. Note that ECGs are time-domain projections of 3-dimensional propagation and conduction of electrical activity of the human heart, while 3-lead VCG presents the added space-time information. Although 12-lead ECG presents such spatiotemporal activity along 12 different perspectives, the loss of spatial information in each temporal ECG tracing undermines the complete picture of $3 \mathrm{D}$ cardiac electrical activity $[9,10]$.

In addition, the blockage of bundle branches can take place at different locations of the heart and with complications such as myocardial infarction [11]. The spatial locations of blocked branches or infarcted lesions lead to the variations of ventricular depolarization and cardiac electrical activity in both space and time. Therefore, there are variations in the QRS morphology of ECG signals from LBBBs and myocardial infarctions. As traditional temporal ECG tracings diminish important spatial information, automated algorithms can be significantly influenced by such an information loss. There is a need to extract useful space-time information from 3-lead VCG and fully exploit the spatiotemporal knowledge to improve the effectiveness of automated algorithms for computerized interpretation of tracings. 
This paper focuses on the unsupervised visualization of patient dissimilarity through pattern matching of VCG signals, as opposed to classification (i.e., supervised learning and training). QRS morphology is commonly used in the electrocardiographic diagnosis of ventricular depolarization such as left bundle branch block (LBBB) and ventricular septal infarction. In this investigation, we hypothesized that pattern matching between QRS loops in 3D VCG improves the grouping of patients whose electrical activity akin to each other, thereby assisting in clinical decision making of LBBB and myocardial infarctions. This is based on the premise that if two subjects share similar space-time trajectories and moving paths in QRS loops of 3D VCG, their electrical activities of ventricular depolarization are also akin to each other. The first step is to develop quantitative measures of pattern similarity and dissimilarity between 3D QRS loops, which should combine pertinent information about the time duration, morphology, notching/slurring, area of QRS loops. Once the patient-to-patient dissimilarity matrix is obtained, the second step is to exploit the acquired knowledge of pattern dissimilarity for optimal clinical decision making. Although 12-lead ECG is standard practice in the state of the art, time-domain interpretation often overlooks the electrical activity in the 3D space. As electrical activity is conducted and propagated in the 3D human heart, new vectorcardiographic methods are needed to characterize the pattern variations of space-time electrical activity in the heart and predict cardiac ailments.

This paper presents new methods to quantitatively measure the pattern dissimilarity of 3D QRS loops, innovatively represent each patient as a node in the network that preserves the patient-to-patient distance matrix, and iteratively self-organize nodes to visualize patient grouping in the network. Experimental results showed that node locations converge as the representation error reaches a stable phase. Spatial coordinates of nodes (or patients) provide a new way to examine the closeness of signal patterns in the space, which is conducive to develop a better understanding of the disease mechanisms (conduction pathway). This pilot study shows strong potentials to establish a large-scale patient dissimilarity network that will enable and assist the visualization of VCG pattern similarity, characterization of VCG pattern variations, cluster subjects with similar cardiac electrical activity in groups, and further predict cardiac ailments.

\section{MATERIALS AND METHODS}

\section{Dataset}

The 12-lead ECGs from 93 patients, equally divided among healthy control, left bundle branch block (LBBB) and antero-septal myocardial infarction (MI) are retrospectively collected from the GE MUSE cardiology management information system at the James A. Haley Veterans' Hospital. This study is performed according to the protocol approved by the Institutional Review Board (IRB) of the same hospital. The dataset is completely de-identified to protect privacy and contains only the electrocardiographic tracing. The demographic information is not included in the dataset in this pilot study. The ECGs have been reviewed by an experienced Cardiologist and the diagnosis confirmed based on accepted criteria. Note that 12-lead ECG is commonly used in 
the clinical practice and is readily accessible from the MUSE cardiology information system. However, 3-lead VCG is not generally recorded and stored in the MUSE system at the James A. Haley Veterans' Hospital. Although prior work shows the need to customize transformation matrices (i.e., from 12-lead ECG to 3-lead VCG) for a subject [12], it is difficult to develop different transformation matrices in the present study. As such, we pre-process the 12-lead ECG printout from GE MUSE Cardiology Information System to obtain the digitized 12 ECG data. Then, a generalized Dower transform was used to synthesize the 3-lead VCG from 12-lead ECG [13]. This brings the flexibility to utilize readily available ECG data in the current practice and avoid the investment of new VCG systems.

Initial evaluation of time-domain $\mathrm{X}, \mathrm{Y}, \mathrm{Z}$ ensembles of 3-lead VCG of the three groups is performed to assess the variations between three datasets. Differences in QRS time duration, morphology, notching/slurring among the groups were evaluated. Fig.1a shows the time-domain X, Y, Z ensembles of 3-lead VCG from 31 subjects of healthy control, LBBB, and MI. It may be noted that there are significant variations within each category of cardiac conditions. Among these three categories, there are also differences in QRS time duration, morphology, notching/slurring, area. However, a single parameter is not sufficient enough to differentiate three categories.
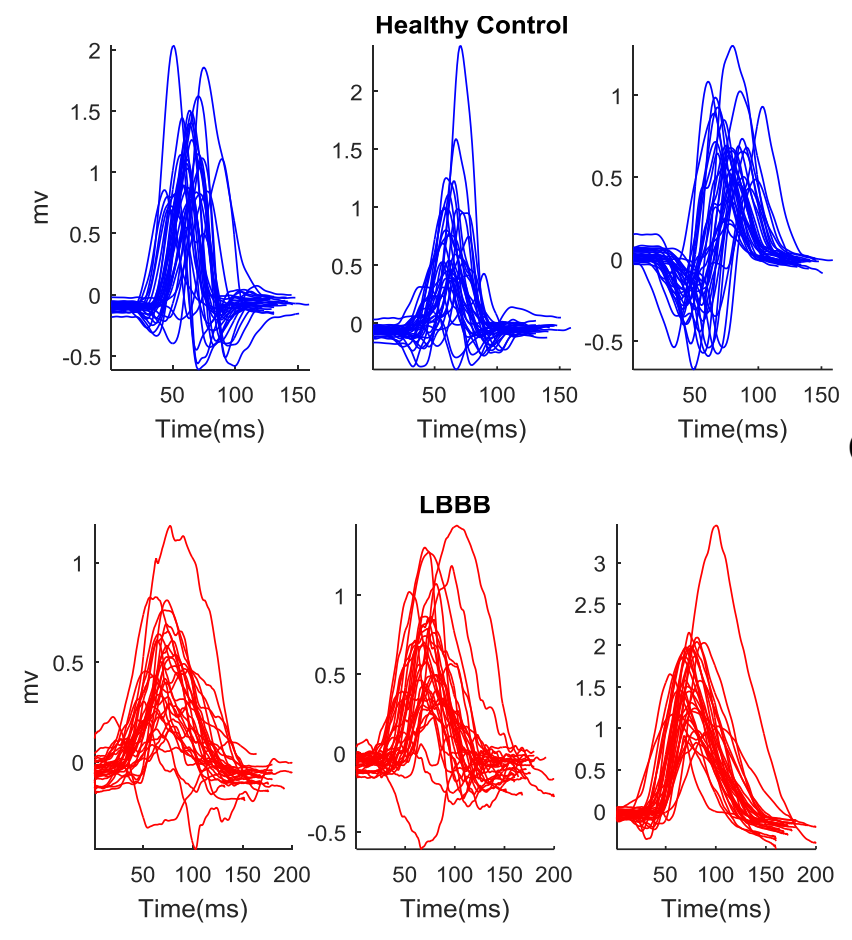

(a)

(b) 

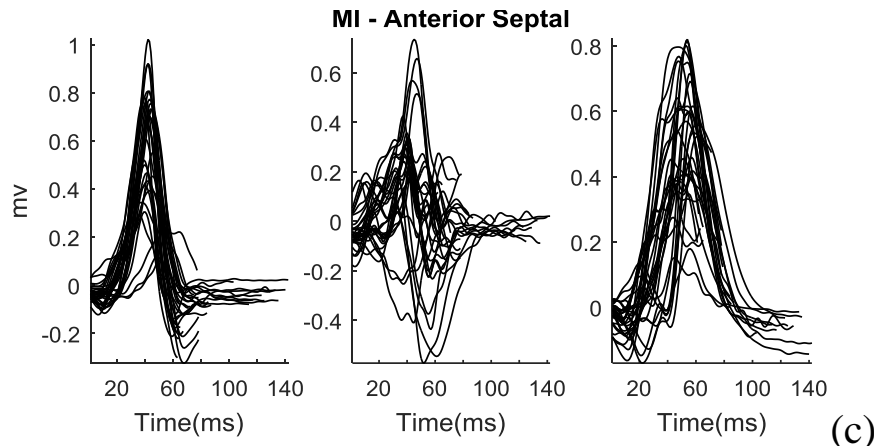

Fig.1. The ensembles of 3-lead VCG, $x y z$ respectively, from 31 subjects of (a) healthy control, (b) LBBB and (c) MI (anterior septal).

Fig. 2 shows the boxplot of QRS time duration for healthy control, LBBB and MI. The red line in the middle of boxplot represents the median, the blue box shows the lower quartile and upper quartile of data distributions, and the black dash lines represent the most extreme values within 1.5 times the interquartile range. Outliers are shown as the red cross in the box plot [14, 15]. The box plot shows that QRS time durations are not normally distributed, but instead yield a skewed distribution. As such, means and standard deviations are not sufficient to fully characterize the distribution of QRS time durations. Note that the mean and standard deviation of QRS time durations are 131.6 $\pm 11.3,165.2 \pm 17.8$, and $90.2 \pm 26.9 \mathrm{~ms}$ for healthy control, LBBB, and anterior-septal MI, respectively. Although means are significantly different, there are overlaps in the box plot of QRS time durations among three categories. While QRS time duration is one parameter describing pattern dissimilarity of VCG, it is challenging to design a composite parameter that integrates the dissimilarity measures of QRS time duration, morphology, notching/slurring, area.

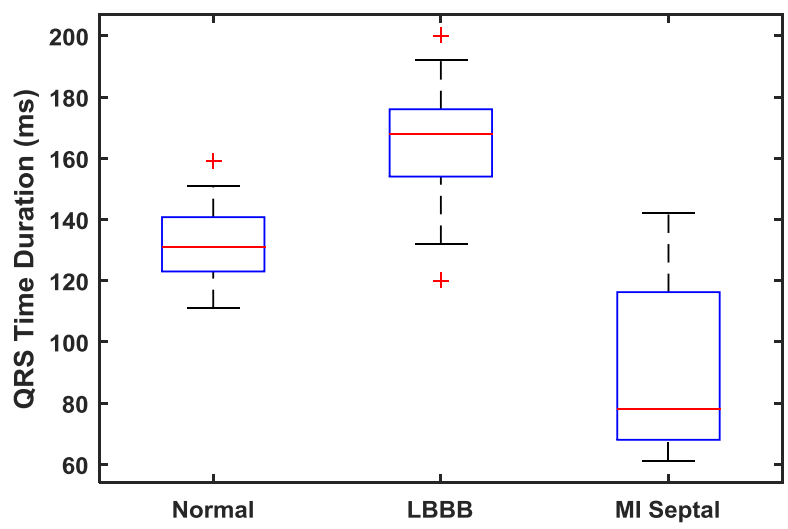

Fig.2. The boxplot of QRS time duration for healthy control, LBBB and MI (anterior septal). 


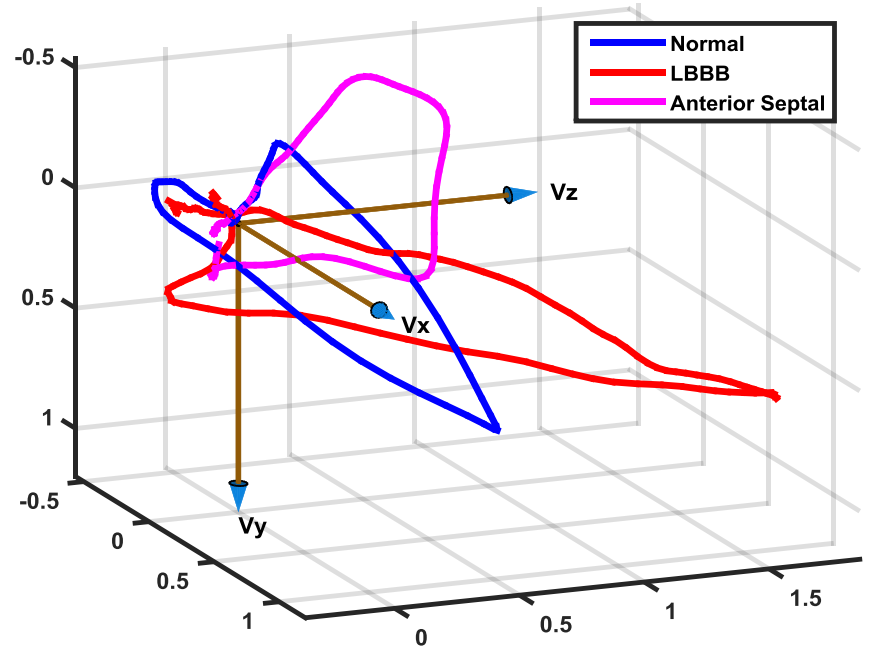

Fig.3. The QRS loops of 3-lead VCG for 3 subjects in the control, LBBB and MI group respectively.

As shown in Fig. 3, QRS loops of 3D VCG yield different spatiotemporal paths for healthy control, LBBB, and MI (anterior septal), including the 3D morphology, area, length (i.e., QRS time duration), angle (i.e., electrical axis). We hypothesized that 3D pattern matching provides dissimilarity information that cannot be otherwise achieved through the parameters extracted in the time-domain ECGs. This is mainly because time-domain ECGs are projections of 3D cardiac electrical activity. Therefore, we propose to develop a composite measure of pattern dissimilarity through pattern matching of 3D VCGs.

\section{Quantitative Measure of Dissimilarity among VCG Waveforms}

Fig. 3 shows that there is significant space-time dissimilarity among QRS loops of 3D VCG of healthy, LBBB and MI-septal subjects. Pattern matching of 3D QRS loops is a new way to identify various types of disorders in the ventricular depolarization. As shown in Fig. 4, two ECG signals are often misaligned because of heart rate variability, phase shift, and discrete sampling. Fig. 4a shows that a bigger dissimilarity will be resulted for two alike ECG signals if time indices are not properly aligned. It is imperative to align the ECG signals in terms of cardiac electrical activity (see Fig. 4b). In other words, such an alignment is critical to compare the corresponding electrical activity of heart chambers. For example, we should compare ventricular depolarization (i.e., QRS loops) for two subjects (or cycles), as opposed to an incorrect comparison between atrial depolarization (P loop) and ventricular depolarization (QRS loop). 

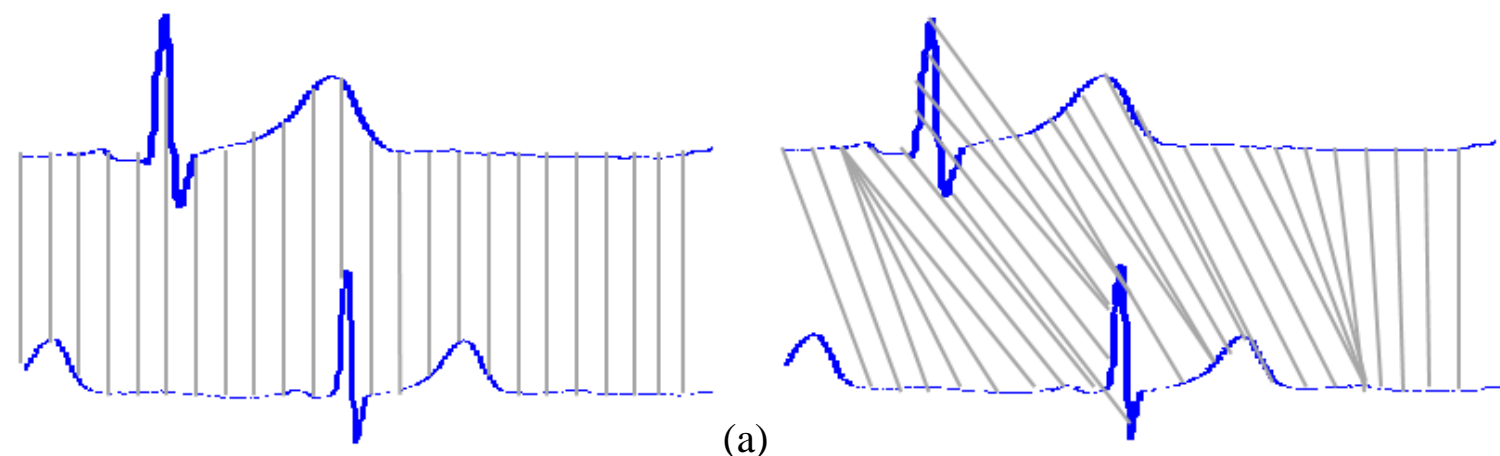

Fig.4. (a) Pattern matching of misaligned ECG signals leads to a bigger dissimilarity for two alike ECG signals; (b) Aligned ECG signals according to electrical activity of heart chambers .

In this investigation, we developed a new warping approach to align 3D VCG vector loops from two subjects and then measure the pattern dissimilarity between them [14]. Note that the alignment of QRS loops in both space and time is critical to compare the electrical activity of ventricular depolarization. If there are two 3D VCG QRS loops $\overrightarrow{\boldsymbol{y}_{1}}(t), t=1, \ldots, N_{1}$ and $\overrightarrow{\boldsymbol{y}_{2}}(t), t=1, \ldots, N_{2}$, their dissimilarity measure is calculated as $\sum_{\left(t_{i}, t_{j}\right) \in \text { path }}\left\|\overrightarrow{\boldsymbol{y}_{\mathbf{1}}}\left(t_{i}\right)-\overrightarrow{\boldsymbol{y}_{\mathbf{2}}}\left(t_{j}\right)\right\|$ by the alignment path. We used the dynamic programming (DP) method to search the optimal path that connect $(1,1)$ to $\left(N_{1}, N_{2}\right)$ in the 2-dimensional lattice. The algorithm starts at the initial condition: $D(1,1)=d(1,1)=\left\|\overrightarrow{\boldsymbol{y}_{1}}\left(t_{1}\right)-\overrightarrow{\boldsymbol{y}_{2}}\left(t_{1}\right)\right\|$ and the search window $\left|t_{i}-t_{j}\right|<r$, and then searches forward iteratively as:

$$
D\left(t_{i}, t_{j}\right)=\min \left(\begin{array}{c}
D\left(t_{i}, t_{j}-1\right)+d\left(t_{i}, t_{j}\right) \\
D\left(t_{i}-1, t_{j}-1\right)+d\left(t_{i}, t_{j}\right) \\
D\left(t_{i}-1, t_{j}\right)+d\left(t_{i}, t_{j}\right)
\end{array}\right)
$$

Finally, the dissimilarity measure of two 3D QRS loops is calculated as:

$$
\Delta\left(\overrightarrow{\boldsymbol{y}_{1}}, \overrightarrow{\boldsymbol{y}_{2}}\right)=\frac{D\left(N_{1}, N_{2}\right)}{N_{1}+N_{2}}
$$

Note that $N_{1}$ and $N_{2}$ are the length of $\overrightarrow{\boldsymbol{y}_{\mathbf{1}}}$ and $\overrightarrow{\boldsymbol{y}_{\mathbf{2}}}$ that are corresponding to the time durations of QRS loops. The distance measure $d\left(t_{i}, t_{j}\right)=\left\|\overrightarrow{\boldsymbol{y}_{\mathbf{1}}}\left(t_{i}\right)-\overrightarrow{\boldsymbol{y}_{\mathbf{2}}}\left(t_{j}\right)\right\|$ captures the morphology, notching/slurring, and area of QRS loops along the alignment path.

\section{Self-organizing Network of VCG QRS Waveforms}

Furthermore, we propose to treat patients as nodes in the network and dissimilarity distance between 3D VCG waveforms as the weights of edges. As shown in Fig. 5a, patient-to-patient dissimilarity matrix provides information pertinent to the pattern variations of 3D VCG QRS loops among patients. It may be noted that each patient's dissimilarity to himself or herself is 0 and dissimilarity measures to all other patients are captured in the matrix. Fig. 5b shows a network of nodes with the dissimilarity distances as the weights of the edges. Ideally, this analysis should optimize the locations of nodes in the network so that node-to-node distances preserve the patient-to-patient dissimilarity measures in the matrix of Fig 5a. 


\begin{tabular}{|c|c|c|c|c|}
\hline \multirow[t]{5}{*}{0.00} & 2.32 & 2.97 & 1.75 & 5.02 \\
\hline & 0.00 & 1.22 & 1.23 & 2.79 \\
\hline & 1.22 & 0.00 & 2.14 & 2.42 \\
\hline & 1.23 & 2.14 & 0.00 & 3.93 \\
\hline & 2.79 & 2.42 & 3.93 & 0.00 \\
\hline
\end{tabular}

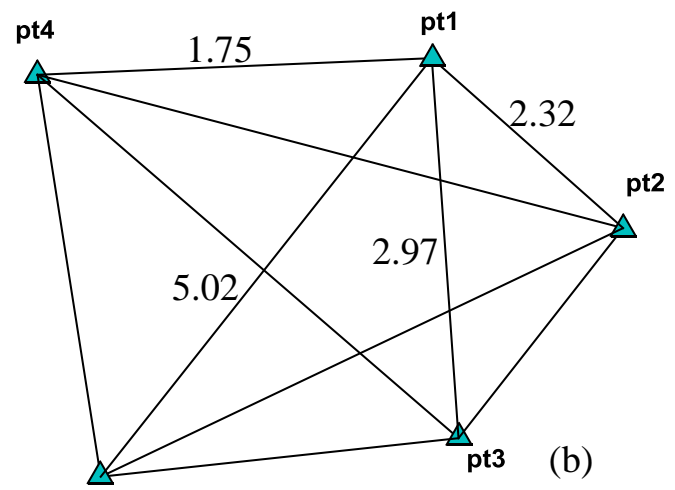

Fig. 5. (a) Dissimilarity matrix among patients; (b) Network pet5 distances that preserve the dissimilarity matrix.

Based on our previous work on self-organizing networks [16, 17], we derive the selforganizing network of patients. Let $\boldsymbol{G}=\{\boldsymbol{V}, \boldsymbol{E}, \boldsymbol{W}\}$ be the directed and weighted network, where $\boldsymbol{V}$ is the set of nodes, $\boldsymbol{E}$ is the set of edges, and $\boldsymbol{W}$ is the set of weights (i.e., dissimilarity measure) on each edge. We utilized the spring-electrical model to assign two forces, i.e., attractive and repulsive forces between nodes. As this network is fully connected, repulsive and attractive forces exist between any pair of nodes (i.e., patients). The repulsive force is defined as

$$
f_{r}(i, j)=-\frac{1}{\|\boldsymbol{s}(i)-\boldsymbol{s}(j)\|^{2}} * e^{\alpha|w(i, j)|}
$$

where $\alpha$ regulates the amplitude of repulsive force, $\mathbf{s}(i)$ and $\mathbf{s}(j)$ are spatial locations of node $i$ and node $j$. The repulsive force is proportional to the edge weight between two nodes (i.e., dissimilarity), because a bigger repulsive force is expected to separate two nodes when they have a bigger weight (i.e., bigger dissimilarity). The attractive force is defined as

$$
f_{a}(i, j)=\|\boldsymbol{s}(i)-\boldsymbol{s}(j)\|^{2} * e^{\frac{\gamma}{|w(i, j)|}}, w(i, j) \neq 0
$$

where $\gamma$ is the natural spring force. The attractive force is inversely proportional to edge weight between two nodes (i.e., dissimilarity), because a bigger attractive force will pull two nodes closer when they have a smaller weight (i.e., smaller dissimilarity). The combined force on a node $i$ is the summation of all repulsive forces and attractive forces on the node:

$$
f(i, \boldsymbol{s}, \alpha, \gamma)=\sum_{i \neq j}-\frac{e^{\alpha|w(i, j)|}}{\|\boldsymbol{s}(i)-\boldsymbol{s}(j)\|^{3}}(\boldsymbol{s}(i)-\boldsymbol{s}(j))+\sum_{i \leftrightarrow j} e^{\frac{\gamma}{|w(i, j)|}}\|\boldsymbol{s}(i)-\boldsymbol{s}(j)\|(\boldsymbol{s}(i)-\boldsymbol{s}(j))
$$

where $i \leftrightarrow j$ means node $i$ and node $j$ are linked, $\boldsymbol{s}(i)-\boldsymbol{s}(j)$ is the force-directional vector, which is separated from $f_{r}(i, j)$ and $f_{a}(i, j)$ to define the direction of combined force $f(i, \boldsymbol{s}, \alpha, \gamma)$. The self-organizing process minimizes the total energy of the network as follows:

$$
\operatorname{Min}_{\boldsymbol{s}}\{\operatorname{Energy}(\boldsymbol{s}, \alpha, \gamma)\}=\operatorname{Min}_{\boldsymbol{s}}\left\{\sum_{i \in N} f^{2}(i, \boldsymbol{s}, \alpha, \gamma)\right\}
$$


As a result, the self-organizing process optimizes spatial locations of network nodes that preserve the edge weights between nodes (i.e., dissimilarity distances between patients). Note that if the parameters $\alpha, \gamma$ are varied, the relative locations of nodes will not change significantly but a similar topology in different scales. See details on the optimization algorithms in our previous work [16, 17]. As the network energy converges, nodes are self-organized in the 3D space and yield a unique topology. Such a self-organizing process drives nodes in the 3D space to preserve the patient-to-patient dissimilarity measures. Our hypothesis is that pattern matching between QRS loops in 3D VCG improves the grouping of patients whose space-time electrical activity akin to each other. This hypothesis will be tested using the dataset of QRS loops of 3D VCG from 93 patients in three categories, namely healthy control, LBBB and MI (anterior septal) that will be detailed in the next section.

\section{RESULTS}

\section{Dissimilarity matrix among VCG waveforms}

Fig. 6 shows the dissimilarity matrix of 93 patients in 3 categories of healthy, LBBB, and MI subjects. The dissimilarity measure is mapped onto a color scale. The small distances are in blue colors with a gradual transition to the large distances in red colors. Note that the dissimilarity matrix is symmetric with on-diagonal squares representing the within-group dissimilarities and off-diagonal squares representing the dissimilarities across groups. As shown in Fig. 6, withingroup distances are smaller than across-group distances. The on-diagonal squares are mainly in blue colors, while off-diagonal squares show light blue and red colors. From the color mapping visualization of the dissimilarity matrix, it is not an easy task to visually discern the patterns for 3 groups. But it may be noted that LBBB group yields the biggest difference from the other two groups. A new method to construct the patient dissimilarity network is urgently needed.

In addition, the dissimilarity matrix cannot be directly used for the purpose of decision making because it records the subject-to-subject dissimilarity instead of predictors for each subject. Such a dissimilarity matrix is a critical component for unsupervised clustering as opposed to supervised classification. Therefore, we propose a new idea to treat patients as nodes in the network and dissimilarity distance as the weights of edges. As a result, we will be able to visualize the clustering or grouping of 93 patients in the network if the network is self-organized according to the edge weights. Spatial coordinates of nodes (or patients) can be used as locators (or features) to assist in the clinical decision making. 


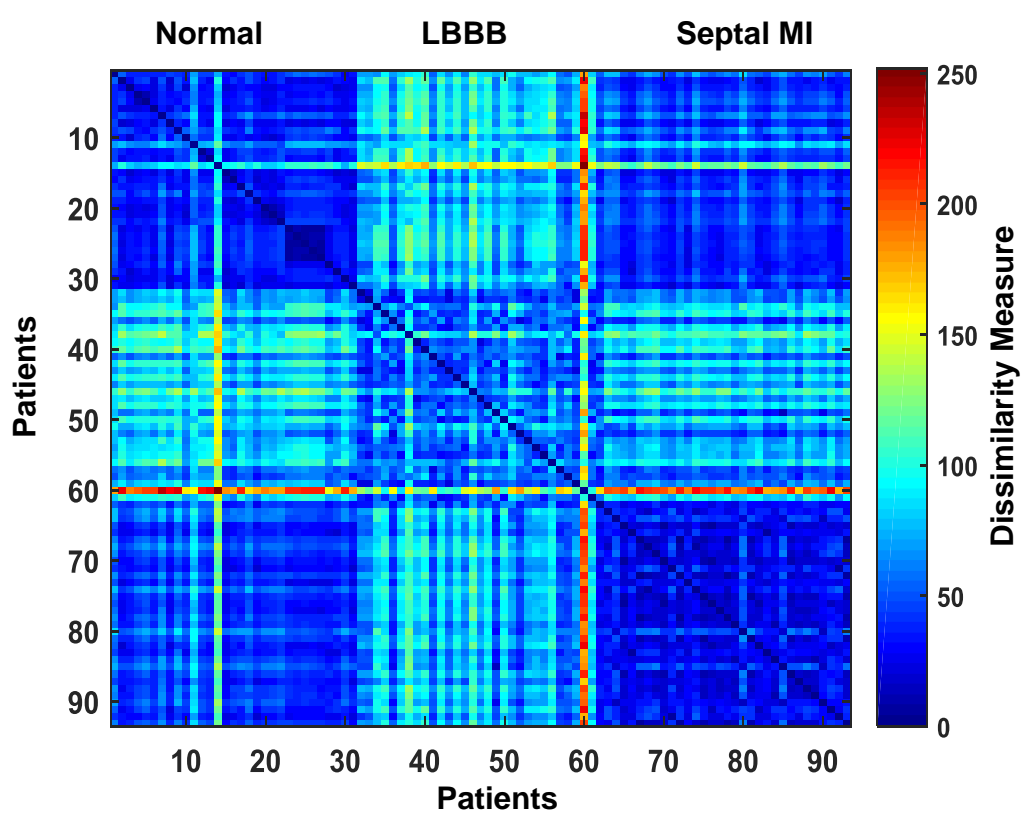

Fig. 6. Color mapping plot of dissimilarity matrix of healthy, LBBB, and MI subjects

\section{Self-organizing visualization of Healthy vs. LBBB subjects}

Fig. 7 shows the scatter plot of nodes of 31 healthy and 31 LBBB subjects in the 3D space. The X-, Y-, Z- scales provide the magnitudes and locations of nodes. The proposed selforganizing algorithm automatically organizes the spatial locations of network nodes based on the edge weights in the patient-to-patient dissimilarity matrix. The edges between nodes are not drawn in the network, because a large number of edges will cover the scatter plot. First, 93 nodes are randomly distributed in the 3D network (see the animation in Fig. 7). It takes approximately 260 iterations ( 37 seconds) for the self-organizing algorithms to reach the stable structure of the network. The performance results and computing time are shown in the animation video that is available at (https://youtu.be/8Kbp3bXL16k). The stable structure means that the spatial locations of nodes converges and do not vary anymore in the 3D space. The iterative variations of the structure of self-organizing networks are shown in the animation link of Fig. 7. It is remarkable that healthy and LBBB subjects yield two distinct clusters in this self-organizing network. This demonstrates that the self-organizing algorithm effectively enables and assists (i) the derivation of stable network structure according to the edge weights (i.e., dissimilarity among patients); (ii) the clustering of patients whose electrical activity akin to each other in the 3D space; (iii) the extraction of pertinent information about cardiac electrical activity in the form of spatial coordinates of network nodes. This algorithm is not only capable of visualizing patients with similar patterns of QRS loops in the 3D space, but also provides a new way to assist in the clinical decision making. 


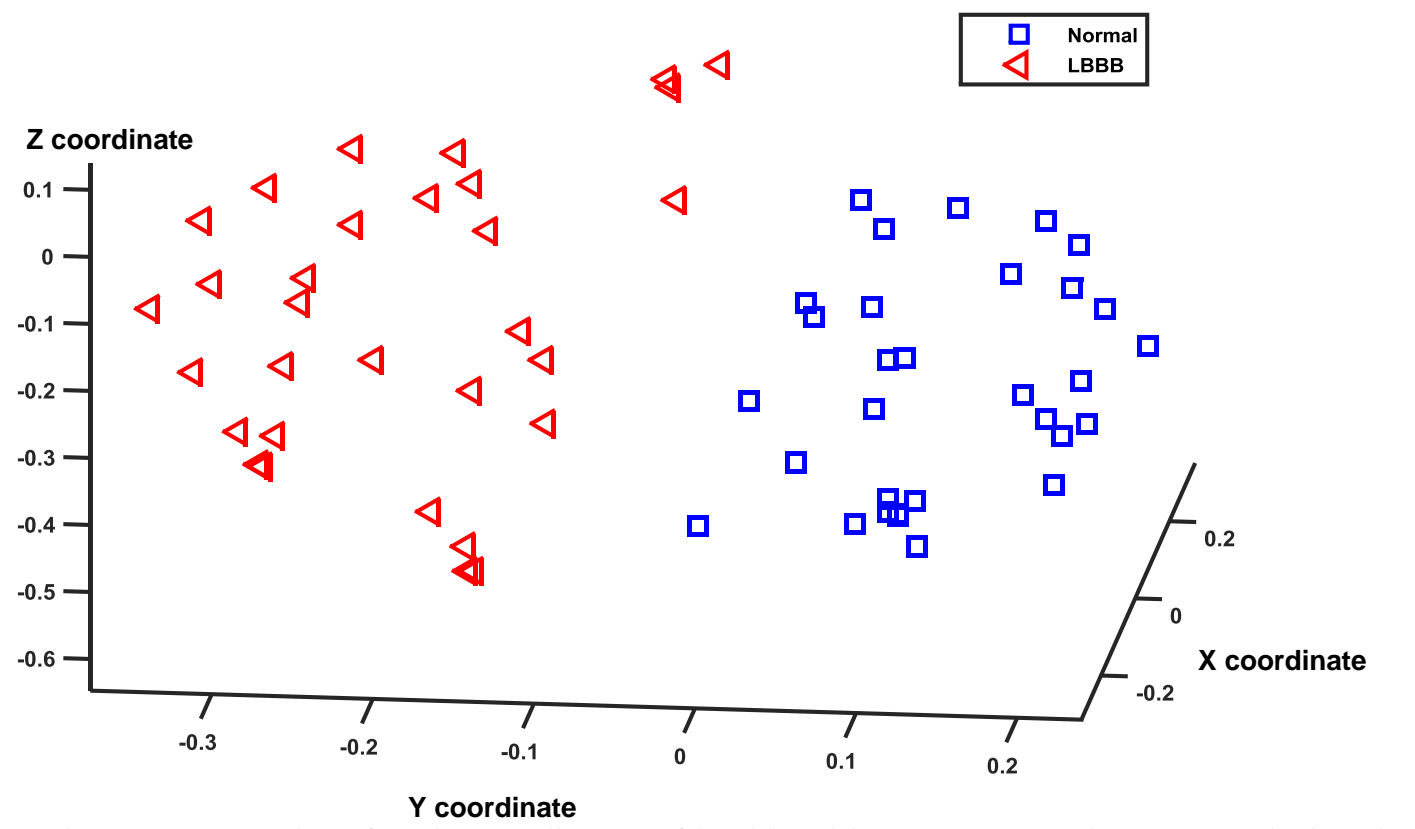

Fig. 7. The 3D scatter plot of node coordinates of healthy (blue squares) and LBBB (red triangles) subjects in the self-organizing network. The animation video is available in this link: https://youtu.be/8Kbp3bXL16k

\section{Self-organizing visualization of LBBB vs. MI Anterior Septal subjects}

Fig. 8 shows the scatter plot of nodes of $31 \mathrm{LBBB}$ and $31 \mathrm{MI}$ anterior septal subjects in the $3 \mathrm{D}$ space. It may be noted that the self-organizing process drives the nodes to automatically cluster according to edge weights (i.e., dissimilarity measures). The $31 \mathrm{LBBB}$ and $31 \mathrm{MI}$ anterior septal subjects yield two distinct clusters when the self-organizing process reaches the stable phase. It is worth mentioning that this self-organizing process is not a supervised classification method, but rather a visualization method to represent pattern similarities and dissimilarities of 3D QRS loops. As such, we can visually discern the patient-to-patient closeness of VCG patterns. 


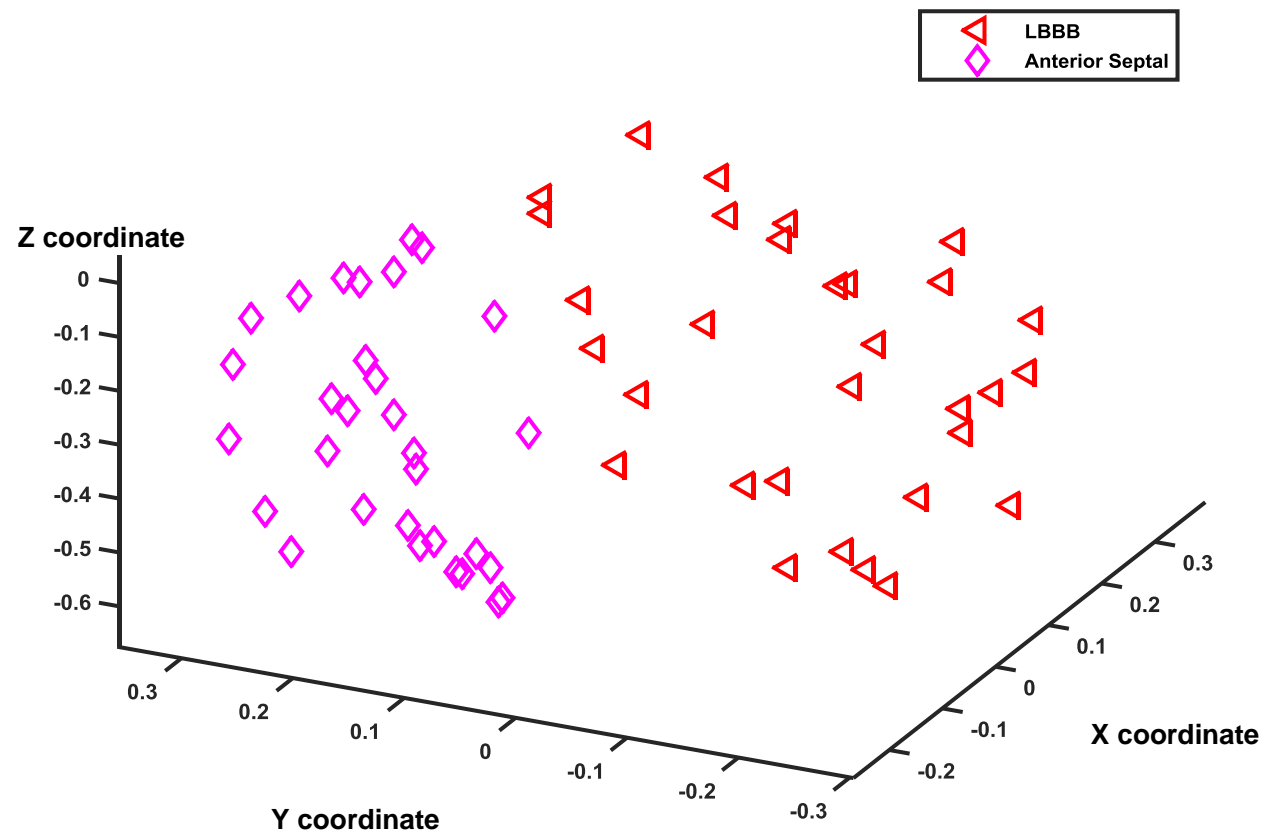

Fig. 8. The 3D scatter plot of node coordinates of LBBB (red triangles) and MI anterior septal (magenta diamond) subjects in the network. The animation video is available in this link: https://youtu.be/y3OEGdEhpgY

\section{Self-organizing visualization of Healthy, LBBB and MI Anterior Septal subjects}

Furthermore, Fig. 9 (a) shows the scatter plot of nodes of 31 healthy, 31 LBBB and 31 MI anterior septal subjects in the 3D space. The self-organization of 3D QRS patterns shows three distinct groups, which are derived on the basis of dissimilarity matrix among patients. There is only one healthy subject falling into the MI anterior septal group. It takes approximately 470 iterations ( 97 seconds) for the self-organizing algorithms to reach the stable structure of the 93patients network. The performance results and computing time are shown in the animation video that is available at (https://youtu.be/RKpVpVlHTGM). It may be noted that 97 seconds includes the visualization and animation. The computing time without animation is about 19.2 seconds for 3 groups of 93 patients.

Fig. 9 (b) shows the iterative variations of network energy for the self-organizing process. Note that the algorithm drives the network energy to keep decreasing and stays stable after 200 iterations. Such experimental results show that (i) each cardiac condition shares similarities of cardiac electrical activity within its own group, but yields bigger dissimilarities from other groups; (ii) such pattern similarities and dissimilarities could be leveraged to visualize the clustering of subjects. However, the proposed self-organizing algorithm enables the pattern matching of VCG signals for visualizing the distribution of subjects and cardiac conditions. 


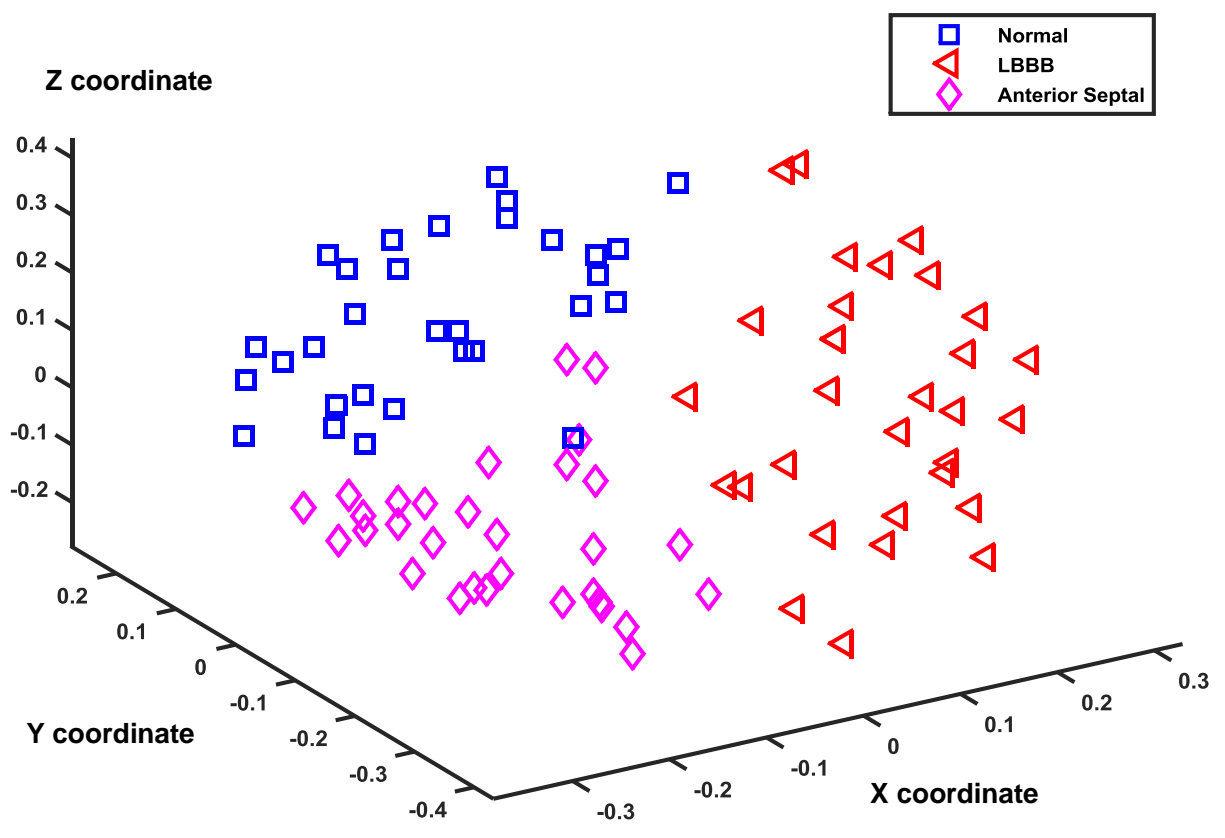

(a)

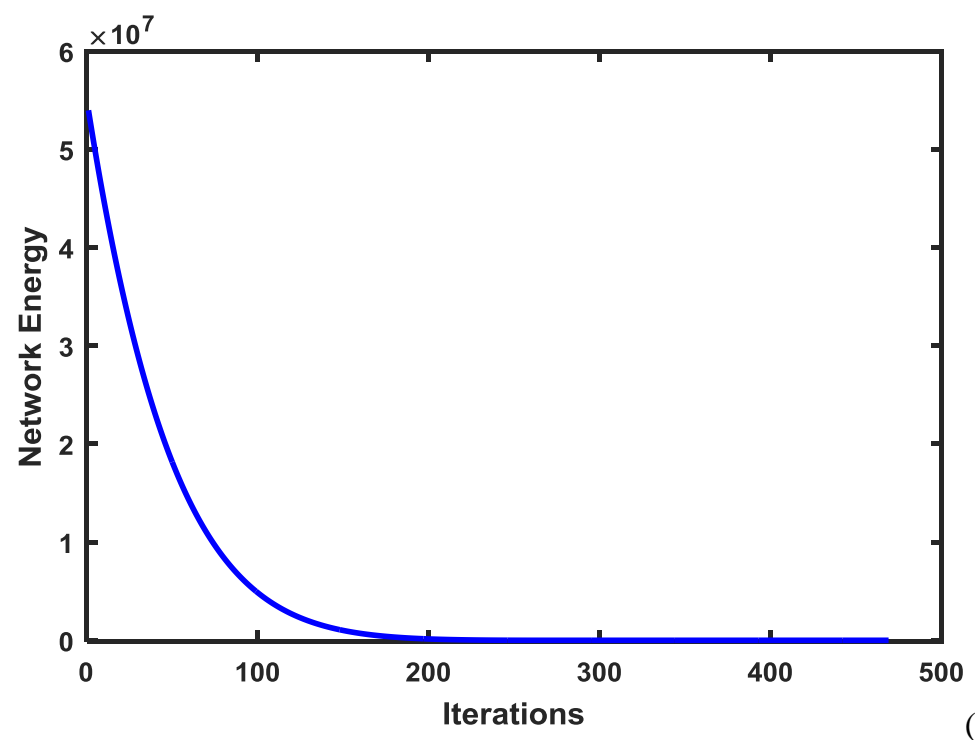

(b)

Fig. 9. (a) The 3D scatter plot of node coordinates of healthy, LBBB and MI anterior septal subjects in the self-organizing network. The animation video is available in this link: https://youtu.be/RKpVpVlHTGM; (b) The iterative variations of network energy for the self-organizing process.

\section{Convergence of self-organizing visualization}

The self-organizing process derives a stable structure of the network by preserving the patient-to-patient edge weights (i.e., dissimilarity matrix), thereby grouping homogeneous patients into sub-network clusters. This self-organizing process will converge if the incremental decrease of network energy $\Delta E$ is smaller than a threshold $\varepsilon$ (i.e., $\Delta E<\varepsilon$ ), where $\Delta E=E(k)-$ $E(k+1)$ is the iterative difference of energy drop and $\varepsilon=0.00001$ is the threshold to detect the stable period in the variations of network energy. At each step, the spatial location of node $i$ will 
move along the direction of combined force $f(i, s, \alpha, \gamma)$ for a magnitude $\vartheta$. Note that the algorithmic convergence is influenced by system parameters $\alpha, \gamma, \vartheta$. The literature showed that the variations of parameters $\alpha, \gamma$ in the attractive and repulsive forces will not change the final structure of network but yield an isomorphic structure only in difference scales [16, 17]. However, the magnitude $\vartheta$ greatly impacts the speed of convergence in the self-organizing algorithm. If the magnitude $\vartheta$ is too large, the convergence speed is fast but the final structure of the network will not be stable. If the magnitude $\vartheta$ is too small, the convergence speed is slow. Therefore, we propose three adaptive schemes to update the magnitude of move step for network nodes: (1) If the network energy continues to decrease for 5 iterations, the magnitude $\vartheta$ will be increased to $\vartheta / 0.9$; ii) If the network energy increases in one iteration, the magnitude $\vartheta$ will be decreased to $0.9 \times \vartheta$; iii) otherwise, the magnitude stays the same.

\section{Supervised classification vs. self-organizing visualization}

The proposed self-organizing methodology is significantly different from traditional classification approaches. The present study focuses on unsupervised learning (or pattern visualization) instead of supervised classification. Unsupervised learning is different from supervised classification because there is no training, validation or test dataset. Supervised classification starts with a training data set. The observations in a training data set are known as training cases. The variables are separated to input predictors and target responses. The purpose of the training data is to generate a classification model that is a concise representation of the association between input predictors and target responses. The predictions made by the classification model on testing data are based on the associations learned from the training data. As shown in Fig. 10, the training data is used to construct a model that associates input predictors to target responses. If there are only two categories of cardiac conditions, the predictions are related to a categorical target variable (i.e., category A or B). In addition, cross validation is often utilized to improve the generalization of classification models that avoid the bias and "overfitting" problems in the learning of association rules. For example, K-fold cross validation partitioned the entire dataset into $\mathrm{K}$ disjoint and equalized folds, in which $\mathrm{K}-1$ folds are used as the training dataset and the remaining 1 fold is for testing. This process is repeated $\mathrm{K}$ times until each of the $\mathrm{K}$ folds is used once for testing [14]. Supervised classification uses three performance measures, i.e., accuracy, sensitivity, and specificity. The accuracy is the ratio of subjects correctly identified in the testing set. Sensitivity measures the proportion of positives which are correctly identified as such, while the specificity measures the proportion of negatives correctly identified. 


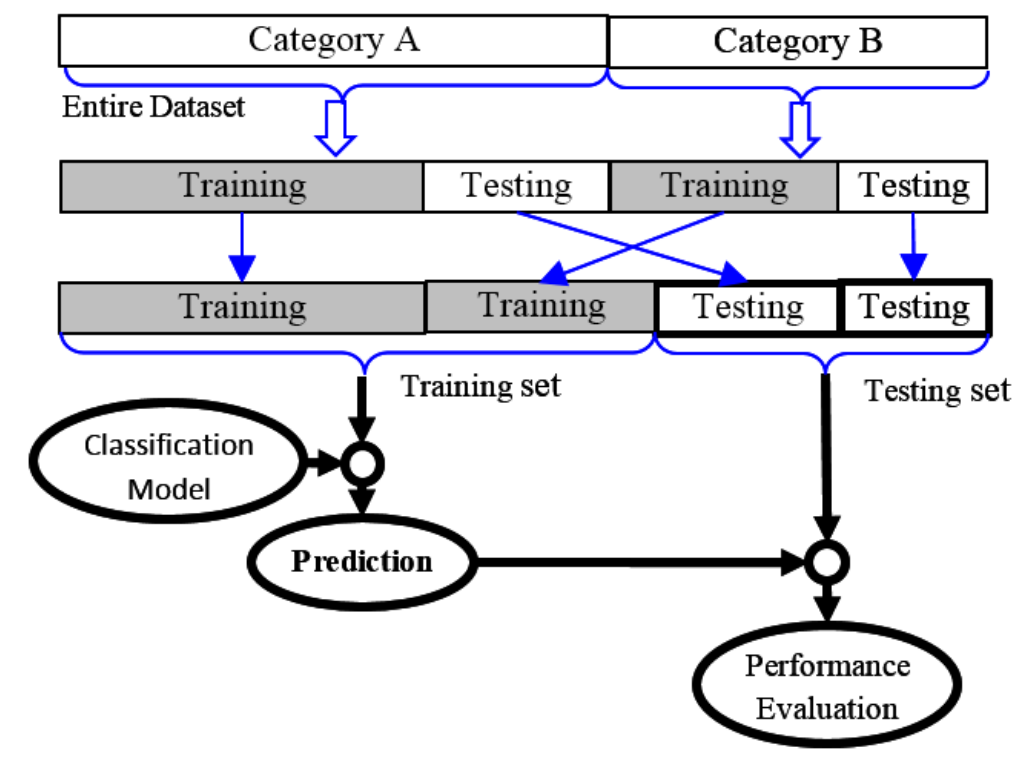

Fig. 10. The framework to learn the supervised classification model

However, this investigation presents a new unsupervised approach, named self-organizing network, to group patients based on pattern similarities in QRS loops of 3D VCG. First, the entire dataset is not separated into training and testing sets. Instead, the self-organizing algorithm is developed to discover the patterns hidden in the entire dataset and then represent patients by a number of clusters based on pattern similarities. The self-organizing visualization is greatly distinguished from traditional supervised classification. However, such self-organizing clustering is also useful as a step in predictive modeling. For example, patients can be clustered into homogeneous groups based on pattern similarities and dissimilarities. Then a predictive model can be built to predict the cluster membership based on the pattern of QRS loops of a new patient.

\section{DISCUSSION}

\section{Predictive values of self-organizing pattern matching}

The present study focuses on the 3D pattern dissimilarity of VCG signals and the selforganizing approach to match $3 \mathrm{D}$ CVG patterns for the construction of patient dissimilarity network. When nodes are self-organized to preserve the patient-to-patient dissimilarities, spatial locations of network nodes are optimized to cluster patients into homogeneous groups. As a result, each node's location in the network is analogous to the locator of each patient in the disease groups. Node locations provide invaluable information that can be used as input variables by the classification model to predict cardiac conditions. In this present study, Fig. 7 shows that healthy and LBBB subjects are clustered into distinct groups that can be easily separated with a linear hyperplane with $100 \%$ accuracy. Similarly, Fig. 8 shows that LBBB and MI anterior septal subjects are also easily separable with a linear hyperplane with $100 \%$ accuracy. Note that sophisticated classification models are not necessary because two clusters are visually separable. 
In addition, Fig. 9 shows that three categories of healthy, LBBB and MI anterior septal subjects are also shown in 3 different clusters. There is only one healthy subject falling into the MI anterior septal group. Experimental results showed that healthy, LBBB and MI anterior septal subjects are discernible using 3D QRS vector loops because the QRS segment is closely pertinent to ailments in ventricular depolarization such as LBBB and ventricular septal MI. However, if the objective is to predict disorders in atrial depolarization or repolarization, it may be necessary to perform pattern matching of the vector loops of $\mathrm{P}$ wave instead of QRS in 3-lead VCG.

Further, this present study considered the offline data of 3-lead VCGs from the database. The developed algorithms are also applicable for the online pattern matching and self-organization of 3-lead VCGs. As shown in Fig. 11, when a new patient is wearing a portable VCG recorder, 3lead VCG recording will be collected in real time. In the practice, each new patient can also enter the clinics for cardiovascular diagnostics. As such, the new patient's pattern dissimilarity will be measured against the database of $N$ patients. Then, a new row and column will be obtained in the dissimilarity matrix, and a new node will be embedded in the high-dimensional network. Finally, classification models can be developed to predict cardiac conditions with node coordinates. Fig. 11 shows the general framework to integrate predictive modeling with self-organizing pattern matching for on-line prediction of cardiac conditions using 3-lead VCGs. The success of such clinical decision support systems depends to a great extent on the available VCG patterns in the database, as well as the computational efficiency of self-organizing algorithms when facing a large number of patients in the database.

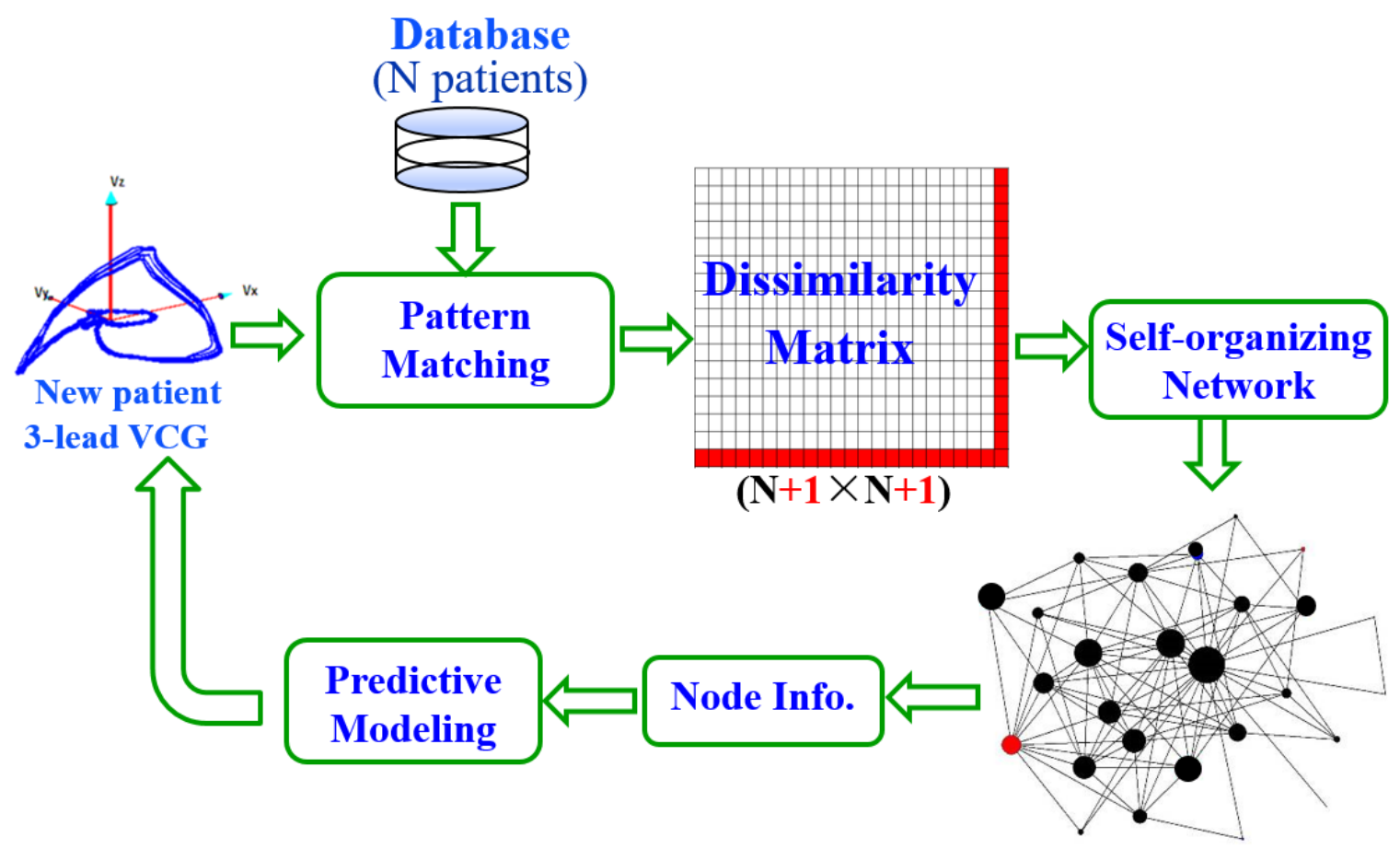

Fig. 11. The framework to integrate predictive modeling with self-organizing pattern matching 


\section{Pattern matching of multi-lead electrocardiograms}

This investigation focuses on the pattern matching of QRS loops in 3-lead VCG, but the algorithm for dissimilarity measure is generally applicable to multi-lead electrocardiograms. However, it may not be necessary to perform pattern matching of 12-lead ECG. Many previous works showed that 12-lead ECG can be derived from 3-lead VCG with a linear transformation matrix (i.e., Dower transform). Over 90\% of ECG energy can be represented in the 3dimensional VCG subspace [18]. Dower transform between 12-lead ECG and 3-lead VCG was demonstrated to preserve clinically useful information pertinent to space-time cardiac electrical activity. Note that 12-lead ECG systems are designed to provide multi-directional views of space-time cardiac electrical activity, while 3-lead VCG views cardiac electrical activity along 3 orthogonal planes of the body, namely, frontal, transverse, and sagittal [19]. As 12-lead ECG has higher dimensionality than 3-lead VCG, this often causes the "curse of dimensionality" in computer analysis. The 3-lead VCG avoids the information loss in 1-lead ECG and overcomes the dimensionality issue in 12-lead ECG [20]. Hence, we propose to use the 3-lead VCG in this present investigation for the pattern match of QRS loops.

This present investigation synthesized 3-lead VCG from 12-lead ECG with the inverse Dower transform. First, we pre-process the 12-lead ECG printout from GE MUSE Cardiology Information System to obtain the digitized 12 ECG data. Second, 12-lead ECG is transformed to 3-lead VCG with the inverse Dower matrix. Finally, 3-lead VCG is utilized for pattern matching and self-organizing visualization. Hence, this paper provides a viable solution for pattern analysis of VCG with the existing 12-lead ECG system, as opposed to purchasing a new set of VCG systems (e.g., Frank orthogonal lead system). On the other hand, this present study shows strong potentials and does not preclude the use of Frank lead system for pattern matching of 3lead VCGs.

\section{Implications for big data and large-scale computing challenges}

Although the present investigation is a pilot study with 93 patients, there are strong implications for large-scale learning of patient groups. It may be noted that pervasive sensing and mobile computing generates big data in the era of Internet of Things (IoT). For example, our prior work developed an IOT system, named Mobile and E-network Smart Health (MESH), integrating mobile ECG sensing with network analytics for smart cardiac monitoring [21]. The presence of big data will indeed provide an unprecedented opportunity. However, there are also great challenges on the computation of dissimilarity measures for a large number of patients and the construction of a large-scale self-organizing network.

Thanks to the next-generation technology of cloud computing, which is a promising and viable solution for big data analytics. The big data challenges can be addressed by leveraging distributed computing resources and accelerating information processing through them. For instance, parallel computing with distributed computers or mobile phones in the internet will assist in the large-scale computation problem. The increasing availability of smartphones 
empowers the collection of ECG/VCG signals using extremely portable sensing devices. The proposed self-organizing network algorithms have great potentials to be integrated with mobile cloud computing to construct a large-scale patient dissimilarity network. The patients will possess a unique location in the network. Sub-network communities are homogeneous disease groups.

In the future, we foresee that the large-scale medical IoT system will be composed of many networked agents at different scales such as ECG sensors, mobile devices, cardiac patients, physicians, emergency centers and hospitals around the world. Such an IoT system enables physicians and cardiologists to access patients' data anytime and anywhere through authorized mobile devices, such as smartphones and tablets. Real-time computation and continual update of patients' conditions in the large-scale IOT system will help make early identification of cardiac diseases and deliver timely treatments to life-threatening disorders.

\section{CONCLUSIONS}

We propose a novel system which identifies pattern differences in 3D VCG loops and uses an automated iterative algorithm to characterize the dissimilarity between electrocardiographic patterns. Very little has been done to perform self-organizing visualization of pattern dissimilarity between VCG signals and derive the patient dissimilarity network. This study represents 93 patients as nodes in a 3-dimensional network. Nodes are iteratively relocated by the developed self-organizing algorithm to preserve the patient-to-patient dissimilarity matrix. Upon the network convergence, node locations of 93 patients automatically emerge into 3 distinct areas of healthy control, LBBB and MI (anterior septal). Self-organizing pattern matching of VCG QRS loops is a data-driven method that effectively groups patients whose ailments in ventricular depolarization are akin to each other. The proposed new approach of selforganizing network is shown to effectively differentiate the variations of 3D patterns of VCG signals and provide patient-to-patient dissimilarity information that cannot be otherwise achieved through the parameters extracted in the time domain. Although medical professionals perform ECG diagnoses every day. However, it is often difficult to visually inspect a large number of ECGs to check the pattern dissimilarity. Most importantly, the visualization of the closeness of signal patterns from multiple patients in the space is conducive to better understand the disease mechanisms (conduction pathway) in addition to diagnostic values. This study provides a new approach to enable the visualization of such information, as opposed to a black-box classification model (difficult to interpret). The clinical utility in future applications of a large-scale patient dissimilarity network will lead to more insights into cardiac pathophysiology. 


\section{ACKNOWLEDGEMENT}

This work is supported in part by the National Science Foundation (CMMI-1646660, CMMI1617148, CMMI-1619648, and IOS-1146882). The authors also thank Harold and Inge Marcus Career Professorship (HY) for additional financial support.

Authors' contribution: HY conceived the study and contributed to the development of spatiotemporal methods and tools, evaluated the data, performed the data analysis, and drafted the manuscript. FML contributed to the design of the study, data collection, clinical interpretation, and revised the manuscript. All authors read and approved the final manuscript.

Conflict of interest statement: No conflicts of interest, financial or otherwise, are declared by the authors. 


\section{REFERENCES}

[1] E. Drazen, N. Mann, R. Borun, M. Laks and A. Bersen. Survey of computer-assisted electrocardiography in the united states. J. Electrocardiol. vol. 21, pp. S98-S104, 1988.

[2] J. Willems, C. Abreu-Lima, P. Arnaud, J. van Bemmel, C. Brohet, R. Degani, B. Denis, J. Gehring, I. Graham, G. van Herpen and ., "The diagnostic performance of computer programs for the interpretation of electrocardiograms," $N$ Engl J Med, vol. 325, no. 25, pp. 1767-1773, 1991.

[3] A. P. Shah and S. A. Rubin, "Errors in the computerized electrocardiogram interpretation of cardiac rhythm," J. Electrocardiol., vol. 40, no. 5, pp. 385-390, 2007.

[4] D. Anh, S. Krishnan and F. Bogun, "Accuracy of electrocardiogram interpretation by cardiologists in the setting of incorrect computer analysis," J. Electrocardiol., vol. 39, no. 3, pp. 343-345, 2006.

[5] M. Westby, I. Bullock, P. N. Cooper and S. Davis, "Transient loss of consciousness - initial assessment, diagnosis, and specialist referral: summary of NICE guidance," BMJ, vol. 341, 2010.

[6] C. J. M. van Deursen, K. Vernooy, E. Dudink, L. Bergfeldt, H. J. G. M. Crijns, F. W. Prinzen and L. Wecke, "Vectorcardiographic QRS area as a novel predictor of response to cardiac resynchronization therapy," J. Electrocardiol., vol. 48, no. 1, pp. 45-52, 2015.

[7] C. J. M. van Deursen, M. Strik, L. M. Rademakers, A. van Hunnik, M. Kuiper, L. Wecke, H. J. G. M. Crijns, K. Vernooy and F. W. Prinzen, "Vectorcardiography as a Tool for Easy Optimization of Cardiac Resynchronization Therapy in Canine Left Bundle Branch Block Hearts," Circulation: Arrhythmia and Electrophysiology, vol. 5, no. 3, pp. 544-552, 2012.

[8] M. Lingman, M. Hartford, T. Karlsson, J. Herlitz, A. Rubulis, K. Caidahl and L. Bergfeldt, "Value of the QRS-T area angle in improving the prediction of sudden cardiac death after acute coronary syndromes," Int. J. Cardiol., vol. 218, pp. 1-11, 2016.

[9] J. L. Willems, E. Lesaffre and J. Pardaens, "Comparison of the classification ability of the electrocardiogram and vectorcardiogram," The American Journal of Cardiology, vol. 59, no. 1, pp. 119-124, 1987.

[10] J. Pettersson, O. Pahlm, L. Sörnmo, W. K. Haisty Jr and L. Edenbrandt, "Increased sensitivity for the diagnosis of healed myocardial infarction using vectorial information in the 12-lead ECG," J. Electrocardiol., vol. 28, no. 3, pp. 169-175, 1995.

[11] T. Q. Le, S. T. S. Bukkapatnam, B. A. Benjamin, B. A. Wilkins, Komanduri and R. Komanduri, "Topology and Random-Walk Network Representation of Cardiac Dynamics for Localization of Myocardial Infarction," IEEE Transactions on Biomedical Engineering, vol. 60, no. 8, pp. 2325-2331, 2013. 
[12] H. Yang, S. T. S. Bukkapatnam, T. Le and R. Komanduri, "Identification of myocardial infarction (MI) using spatio-temporal heart dynamics," Medical Engineering \& Physics, vol. 34, no. 4, pp. 485-497, 2011.

[13] D. Dawson, H. Yang, M. Malshe, S. T. S. Bukkapatnam, B. Benjamin and R. Komanduri, "Linear affine transformations between 3-lead (Frank XYZ leads) vectorcardiogram and 12lead electrocardiogram (ECG) signals," Journal of Electrocardiology, vol. 42, no. 6, pp. 622-630, 2009.

[14] H. Yang, C. Kan, Y. Chen and G. Liu, "Spatiotemporal differentiation of myocardial infarctions," IEEE Transactions on Automation Science and Engineering, vol. 10, no. 4, pp. 938-947, 2013.

[15] G. Liu and H. Yang, "Multiscale adaptive basis function modeling of spatiotemporal cardiac electrical signals," IEEE Journal of Biomedical and Health Informatics, vol. 17, no. 2, pp. 484-492, 2013.

[16] H. Yang and G. Liu, "Self-organized topology of recurrence-based complex networks," Chaos, vol. 23, no. 4, pp. 043116, 2013.

[17] G. Liu and H. Yang, "A self-organizing method for predictive modeling with highlyredundant variables," in Proceedings of 2015 IEEE International Conference on Automation Science and Engineering (CASE), Gothenburg, Sweden, 2015, pp. 1084-1089.

[18] J. Malmivuo and R. Plonsey, Bioelectromagnetism: Principles and Applications of Bioelectric and Biomagnetic Fields. USA: Oxford University Press, 1995.

[19] H. Yang, "Multiscale Recurrence Quantification Analysis of Spatial Cardiac Vectorcardiogram (VCG) Signals," Biomedical Engineering, IEEE Transactions on, vol. 58, no. 2, pp. 339-347, 2011.

[20] H. Yang, S. T. S. Bukkapatnam and R. Komanduri, "Spatio-temporal representation of cardiac vectorcardiogram (VCG) signals," Biomedical Engineering Online, vol. 11, no. 2, pp. 16-30, 2012.

[21] C. Kan, Y. Chen, F. M. Leonelli and H. Yang, "Mobile sensing and network analytics for realizing smart automated systems towards health internet of things," in Proceedings of 2015 IEEE International Conference on Automation Science and Engineering (CASE), Gothenburg, Sweden, 2015, pp. 1072-1077. 